\title{
Morphological Features of Minimal Change Disease and Focal Segmental Glomerulosclerosis Using Repeat Biopsy and Parietal Epithelial Cell Marker
}

\author{
Tomo Suzukia, b Kaori Kohatsu ${ }^{a}$ Wei Han ${ }^{a}$ Shiika Watanabe ${ }^{a}$ Koichi Yahagi ${ }^{c}$ \\ Mayumi Nakata ${ }^{a}$ Toshiharu Ueno ${ }^{a, d}$ Daisuke Ichikawa ${ }^{a}$ Naohiko Imai ${ }^{a}$ \\ Sayuri Shirai ${ }^{c}$ Junki Koike ${ }^{\mathrm{e}}$ Yugo Shibagakia $^{\mathrm{a}}$ \\ a Division of Nephrology and Hypertension, Department of Internal Medicine, St. Marianna University School of

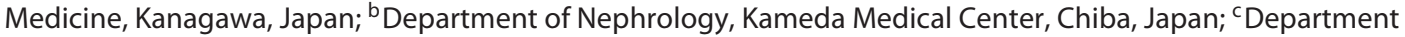 \\ of Nephrology and Hypertension, Yokohama City Seibu Hospital, St. Marianna University School of Medicine, \\ Yokohama, Japan; ${ }^{\mathrm{d}}$ Department of Dialysis, Nakayama Station Clinic, Kanagawa, Japan; ${ }^{\mathrm{e} D e p a r t m e n t}$ of Diagnostic \\ Pathology, Kawasaki Municipal Tama Hospital, Kawasaki, Japan
}

\section{Keywords}

Biopsy · Epithelial cells · Focal segmental glomerulosclerosis · Minimal change disease · Parietal epithelial cell marker

\begin{abstract}
Introduction: Minimal change disease (MCD) and primary focal segmental glomerulosclerosis (FSGS) are representative podocyte diseases. The clinical cause of MCD and FSGS has not been clearly elucidated yet. However, it is important to distinguish MCD and FSGS because their prognoses and responses to treatment are quite different. Objective: This study aimed to examine whether parietal epithelial cell (PEC) marker and repeat biopsy are useful for diagnosing primary FSGS. Methods: Clinicopathological features of 17 patients with the nephrotic syndrome, who underwent kidney biopsy $\geq 2$ times from 1975 to 2017, and had MCD or FSGS were analyzed using PAX8. We defined patients with PAX8+ cells as PAX8+ and the remainder as PAX8- patients. Three cases of sample insufficiency and 1 non-steroid-resistant or frequently relapsing case indicated for repeat biopsy were ex-
\end{abstract}

cluded. Results: Among the 13 patients studied, 4 were PAX8+ and 9 were PAX8- (median age: 41 and 46 years, respectively, at first biopsy). PAX8+ and PAX8- patients showed no significant differences in clinical data and histological diagnosis except for a significant difference in histological diagnosis at the second biopsy. The number of PAX8+ patients increased to 6 . Unlike the first biopsy results, FSGS was present in 5 of 6 (83.3\%) PAX8+ patients; MCD occurred in all 7 (100\%) PAX8- patients. Three of 6 (50.0\%) PAX8+ patients undergoing repeat biopsy were steroid resistant; no (0\%) PAX8- patient was steroid resistant. All cases of final FSGS diagnosis were PAX8+ at the first or second biopsy. Only 1 PAX8+ MCD patient was steroid resistant. All PAX8$M C D$ patients were frequently relapsing. Conclusions: More PAX8+ patients were diagnosed with FSGS than PAX8- patients. Clinical presentation of MCD in PAX8- patients was frequently relapsing. $\mathrm{PEC}$ marker staining in patients with the nephrotic syndrome, e.g., MCD, may help to diagnose FSGS.
(C) 2020 The Author(s) Published by S. Karger AG, Basel

\begin{tabular}{ll}
\hline KARGER & @ 2020 The Author(s) \\
& Published by S. Karger AG, Basel Openger \\
karger@karger.com & This article is licensed under the Creative Commons Attribution- \\
www.karger.com/kdd & NonCommercial-NoDerivatives 4.0 International License (CC BY- \\
& NC-ND) (http://www.karger.com/Services/OpenAccessLicense). \\
& Usage and distribution for commercial purposes as well as any dis- \\
tribution of modified material requires written permission.
\end{tabular}

Tomo Suzuki, MD, PhD

Department of Internal Medicine

St. Marianna University School of Medicine, 2-16-1 Sugao

Miyamae-ku, Kawasaki, Kanagawa 216-8511 (Japan)

E-Mail t2suzuki@marianna-u.ac.jp 


\section{Introduction}

Minimal change disease (MCD) and primary focal segmental glomerulosclerosis (FSGS) are representative podocyte diseases [1]. MCD and primary FSGS present clinically as acute-onset nephrotic syndrome. In addition, the clinical causes of MCD and FSGS are often ambiguous. However, it is important to distinguish MCD and FSGS because they have different prognoses and respond differently to treatment.

The morphological difference between MCD and FSGS is the presence of segmental sclerotic lesions in light microscopy. Thus, whether a case is MCD or FSGS is difficult to diagnose if kidney specimens are scarce. In addition, sclerotic lesions characteristic of FSGS are more commonly found in the corticomedullary area $[1,2]$.

Differentiating MCD from FSGS is often difficult if the specimen is not sampled from the kidney core region. Therefore, we sometimes considered performing repeat biopsy when we suspected FSGS even if the first biopsy indicated MCD. In an experiment of collapsing FSGS, parietal epithelial cells (PEC) increased with podocyte loss $[3,4]$. PEC activation markers are increased in early FSGS recurrence during transplantation [5]. Recently, detection of activated PEC on the glomerular tuft was reported useful to distinguish early FSGS from MCD in humans [6]. However, it is uncertain whether patients with activated PEC for early FSGS and MCD should be diagnosed with FSGS because early FSGS did not show the classical sclerotic lesions, that is, disappearance of cells (endothelial cells and podocytes) and increase in extracellular matrix. Thus, this study aimed to examine whether a PEC marker is useful for diagnosing FSGS and whether a patient with the nephrotic syndrome and PEC marker (PAX8) presented FSGS lesion by repeat biopsy.

\section{Materials and Methods}

\section{Patients}

We conducted a cross-sectional, retrospective study at a single center. The subjects were 17 patients with MCD or FSGS with the nephrotic syndrome, who underwent kidney biopsy $\geq 2$ times from January 1, 1975, to July 31, 2017. Cases of sample insufficiency ( $\leq 5$ glomeruli; $n=3$ ) and those indicated for repeat biopsy who were not steroid resistant or frequently relapsing $(n=1)$, were excluded, leaving a total of 13 patients. Transplant patients were also excluded. A steroid-resistant case is defined as a case in which a sufficient dose of steroid treatment fails to achieve complete remission or incomplete remission 1 month after the initiation of treatment, and a frequently relapsing case is defined as $\geq 2$ relapse events within 6 months [7].
Clinical data and reasons of repeat biopsy were obtained from electronic medical records and paper charts. The first and second biopsies of the PAX8+ and PAX8- groups were compared. The reasons for repeat biopsy were being steroid resistant or frequently relapsing.

\section{Pathological Evaluations}

For light microscopy, samples were stained with hematoxylin and eosin (HE), periodic acid-Schiff (PAS), Masson's trichrome (MT), and Jones' methenamine silver (PAM). Paraffin-embedded material was sectioned at $2-3 \mu \mathrm{m}$ for HE, PAS, and MT staining. For PAM staining, thin sections $(1 \mu \mathrm{m})$ were used. A renal pathologist and nephrologist provided the diagnosis of either MCD or FSGS based on the staining results. Polyclonal PAX8 antibody (Proteintech, dilution 1:50, catalog 10336-1-AP) was used to evaluate activated PEC from paraffin sections. The experiments were carried out as we previously described [4]. The number of PAX8stained cells in the nucleus was counted.

\section{Statistical Analysis}

Continuous variables are presented as medians with interquartile range (IQR [25th-75th percentile]). Categorical variables are presented as numbers and percentages. All values were expressed as means \pm SD or medians plus IQR. Statistical significance was set at $p<0.05$. Patient groups were compared by Student's $t$ test and Pearson's $\chi^{2}$ test. Statistical analyses were performed with JMP 11.0.0 (SAS Institute Inc., Cary, NC, USA).

\section{Results}

Figure 1 shows a light-microscopic image. Early FSGS at the first biopsy is shown in Figure 1a. This segmental lesion constricted the capillary lumen indicating endotheliosis, with slight proliferation of the epithelial cell around the lesions. However, this lesion is not clearly found to have sclerosis, which suggests the difficulty in diagnosing FSGS based on the appearance of the glomerulus. In fact, we diagnosed MCD at the first biopsy. PAX8+ cells proliferated at the tip site (Columbia classification, tip variant) in another glomerulus (Fig. 1b). Then, at the second biopsy, we found segmental sclerotic lesions in the representative glomerulus (Fig. 1c). Moreover, PAX8+ cells proliferated in the sclerotic lesion (Fig. 1d). Thus, we have only evaluated PAX8+ cell and sclerotic lesions during the repeat biopsy.

The clinical characteristics of the patients at the first biopsy are shown in Table 1. All clinical presentations of the population in our cohort are those of the nephrotic syndrome with edema. For the initial treatment of the nephrotic syndrome, all patients were on glucocorticoids (oral prednisolone $0.8-1.0 \mathrm{mg} / \mathrm{kg}$ with or without intravenous methylprednisolone 0.5 or $1 \mathrm{~g}$ per day for 3 days as steroid pulse therapy). We did not have a treatment 
Fig. 1. Kidney biopsy specimen. a PAS staining of glomeruli shows segmental adhesion and mild epithelial cell hyperplasia at the first biopsy. b PAX8 staining of the glomeruli with no sclerosis shows hyperplasia of positive epithelial cells at the tubular pole (red arrow). c PAS staining of the glomeruli shows segmental sclerosis at the second biopsy. d PAX8 staining of the glomeruli shows infiltration of positive cells at sclerotic lesion (red arrow).
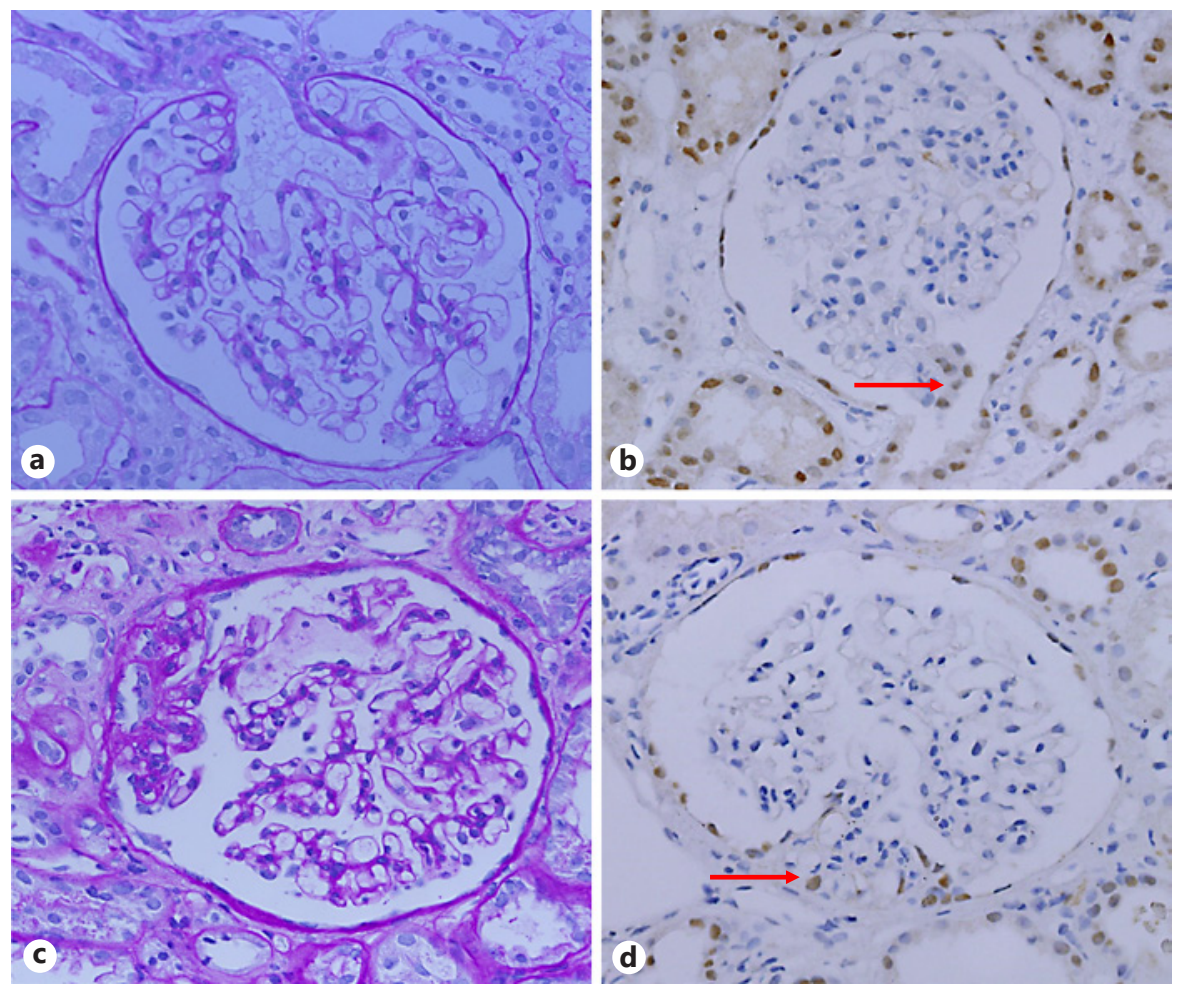

Table 1. Baseline characteristics at the first biopsy

\begin{tabular}{lccc}
\hline & PAX8+ $(n=4)$ & PAX8- $(n=9)$ & $p$ value \\
\hline Males, $n$ (\%) & $3(75.0)$ & $7(77.8)$ & 0.91 \\
Medians (IQR) & & & \\
$\quad$ Age, years & $41(25-67)$ & $46(27-57)$ & 0.53 \\
Serum creatinine, mg/dL & $1.15(0.96-2.05)$ & $1.20(0.85-135)$ & 0.47 \\
Serum albumin, g/dL & $2.3(1.8-3.1)$ & $2.0(1.9-2.6)$ & 0.66 \\
Serum total cholesterol, mg/dL & $386(269-503)$ & $406(287-524)$ & 0.72 \\
$\quad$ Duration until repeat biopsy, months & $18(13-49)$ & $26(9-84)$ & 0.48 \\
\hline
\end{tabular}

protocol for immunosuppressive agents (e.g., calcineurin inhibitor). Among 13 patients, 4 were PAX $8+$ and 9 patients were PAX8-. The median age of the patients was 41 and 46 years, respectively, at the first biopsy. Moreover, no differences were found between PAX8+ and PAX8patients regarding other clinical data (sex, renal function, serum albumin, and serum total cholesterol) and duration to the second biopsy.

Histological diagnoses and evaluation of PAX8+ cells at the first biopsy are summarized in Table 2. Most of the PAX8- patients had a histological diagnosis of MCD (88.9\%), but no differences were found in the histological diagnosis and repeat biopsy reasons between the PAX8+ and PAX8-groups. Further, results of the second biopsy

MCD and FSGS Using Repeat Biopsy and PEC Marker are shown in Table 3. The number of PAX8+ patients increased from 4 to 6 . Unlike the results of the first biopsy, a significant difference was found in the histological diagnosis at the second biopsy. Of the 6 PAX $8+$ patients, 5 (83.3\%) had FSGS, whereas all PAX8- patients had MCD $(7 / 7,100 \%)$. Regarding the Columbia classification of FSGS, 1 had a tip variant, and 5 were not otherwise specified. As regards the reason for repeat biopsy, a significant difference was found between PAX8+ and PAX8- patients. Among the 6 PAX8+ patients, 3 (50.0\%) were steroid resistant, but no PAX8 - patient was steroid resistant $(0 \%)$. In addition, the rate of PAX8+ glomeruli (8.8\%) at the second biopsy was higher than that at the first biopsy (4.1\%) (Tables 2, 3). 
Table 2. Histological characteristics and reason for repeat biopsy at the first kidney biopsy (1st $\mathrm{KBx}$ )

\begin{tabular}{lccc}
\hline & $\begin{array}{c}\text { PAX8+ } \\
(n=4)\end{array}$ & $\begin{array}{c}\text { PAX8- } \\
(n=9)\end{array}$ & $p$ value \\
\hline MCD at 1st KBx, $n(\%)$ & $3(75.0)$ & $8(88.9)$ & 0.52 \\
FSGS at 1st KBx, $n(\%)$ & $1(25.0)$ & $1(11.1)$ & 0.15 \\
Median number of glomeruli at 1st KBx (IQR) & $23(13-45)$ & $17(12-28)$ & 0.91 \\
PAX8+ glomeruli/all glomeruli (SD), \% & $7.4(4.1)$ & $0(0)$ & $7(77.8)$ \\
Reason for repeat biopsy, $n(\%)$ & $3(75.0)$ & $2(22.2)$ & 0 \\
$\quad \begin{array}{l}\text { Frequently relapsing } \\
\text { Steroid resistant }\end{array}$ & $1(25.0)$ & & \\
\hline
\end{tabular}

Table 3. Histological characteristics and reason for repeat biopsy at the second kidney biopsy (2nd KBx)

\begin{tabular}{lccr}
\hline & $\begin{array}{c}\text { PAX8+ } \\
(n=6)\end{array}$ & $\begin{array}{l}\text { PAX8- } \\
(n=7)\end{array}$ & $p$ value \\
\hline MCD at 2nd KBx, $n(\%)$ & $1(16.7)$ & $7(100)$ & $<0.01$ \\
FSGS at 2nd KBx, $n(\%)$ & $5(83.3)$ & $0(0)$ & 0.19 \\
Median number of glomeruli at 2nd KBx (IQR) & $20(13-22)$ & $12(9-25)$ & $0(0)$ \\
PAX8+ glomeruli/all glomeruli (SD), \% & $9.0(8.8)$ & $7(100)$ & 0.03 \\
Reason for repeat biopsy, $n$ (\%) & $3(50.0)$ & $0(0)$ & \\
$\quad$ Frequently relapsing & $3(50.0)$ & & \\
Steroid resistant & &
\end{tabular}

Finally, we examined the change in the histological diagnosis based on the reason for repeat biopsy, which ranged from being frequently relapsing to being steroid resistant (Table 4). Patients with a final diagnosis of FSGS were PAX8+ at the first or second biopsy. Further, 3 of 10 patients who were frequently relapsing had FSGS. In addition, 2 of 10 patients diagnosed with MCD were found to have FSGS among patients who had frequently relapsing symptoms. Thus, 2 of 3 PAX $8+$ patients who had MCD at the first biopsy were considered to have FSGS. Only 1 MCD patient was PAX8+, but the reason of the repeat biopsy was the steroid-resistant status. MCD patients who were PAX8- were all frequently relapsing.

\section{Discussion}

In this study, we report a cohort of 13 MCD or FSGS patients whose diagnoses were based on PEC marker and repeat biopsy results. Our study has 2 novel insights. First, we could not observe FSGS lesions at the first biopsy in patients with PAX8+ nephrotic syndrome, but the second biopsy could have confirmed FSGS. Second, PAX8- MCD cases were all frequently relapsing at the second biopsy.
Table 4. Histological change from the first to the second kidney biopsy

\begin{tabular}{lll}
\hline $\begin{array}{l}\text { Diagnostic change } \\
(1 \mathrm{st} \rightarrow \text { 2nd KBx) }\end{array}$ & $\begin{array}{l}\text { Frequently } \\
\text { relapsing, } n(n=10)\end{array}$ & $\begin{array}{l}\text { Steroid resistant, } \\
n(n=3)\end{array}$ \\
\hline $\mathrm{MCD} \rightarrow \mathrm{MCD}$ & $7(\mathrm{PAX}-8-)$ & $1(\mathrm{PAX}-8+)$ \\
$\mathrm{FSGS} \rightarrow$ FSGS & $1(\mathrm{PAX}-8+)$ & $1(\mathrm{PAX}-8+)$ \\
$\mathrm{MCD} \rightarrow$ FSGS & $2(\mathrm{PAX}-8+)$ & $1(\mathrm{PAX}-8+)$ \\
\hline
\end{tabular}

PAX8+, first biopsy or second biopsy.

MCD and FSGS are known nephrotic syndromes. Some circulating factors were considered one of the causes of primary FSGS [8]. Serum soluble urokinasetype plasminogen activator receptor was cited as a powerful candidate indicating primary FSGS [9]. However, circulating factors were clearly unknown yet. Therefore, to make a diagnosis of MCD or FSGS, kidney biopsy is necessary. Some MCDs, especially its tip lesion, will shift to FSGS [10]. However, it is difficult to judge whether there was a change from MCD to FSGS or whether it was FSGS from the beginning based on a single kidney biopsy. Ahmad and Tejani [11] reported that 14 of 49 patients with 
childhood MCD were found to have FSGS during repeat biopsy. However, this study had not examined PEC markers, and in some of the 14 individuals FSGS was possibly missed in the first biopsy due to a sampling error or earlystage FSGS. In our study, we observed the transition in the diagnosis from MCD to FSGS in 2 of 3 PAX8+ patients on repeat biopsy. Further, 5 of 6 PAX8+ patients had FSGS. Considering our results, we were possibly not able to provide the most appropriate diagnosis because of possible sampling error or early-stage FSGS lesions.

Clinical presentation is important in the management of the nephrotic syndrome. Clinical presentations are categorized into frequently relapsing and steroid sensitive, for example. In general, we recognized that MCD was frequently relapsing, and FSGS has steroid-resistant presentation. In a previous study of 85 children with steroidsensitive nephrotic syndrome, 1 had FSGS [12]. In addition, relapse is not an indication for biopsy because steroid sensitivity is more important than histology, and it is the major determinant of prognosis in the childhood nephrotic syndrome [13]. However, these data are scarce in adults. In our study, 3 of 10 patients with frequently relapsing disease had FSGS (30\%). These data might be impressive for clinicians. Considering the examination of the PEC marker, all PAX8- patients with frequently relapsing presentations had MCD. In addition, 5 of 6 PAX8+ patients had FSGS. Further, the remaining PAX8+ patient was steroid resistant. In our adult patients, PAX positivity is possibly associated with FSGS and steroid resistance.

Our results have 2 clinical implications. First, our study suggests performing repeat biopsy. When the clinician is suspicious regarding the MCD diagnosis, they should consider a repeat biopsy, especially in steroid-resistant cases. However, our study showed that 3 of 10 (30\%) frequently relapsing patients had FSGS. In general, the complications of a percutaneous renal biopsy are scarce (approximately 1\%) in adults and elderly $[14,15]$. However, this rate was not low in medical procedures for internal medicine. If we use a PEC marker for the MCDsuspected nephrotic syndrome, the frequency of repeat biopsy may be reduced. Second, the treatment should be associated with the results of the accurate histological diagnosis. Recently, 2 studies reported about the effectiveness of rituximab for childhood-onset frequently relapsing nephrotic syndrome (mainly MCD) and childhoodand adult-onset steroid-dependent nephrotic syndrome $[16,17]$. On the contrary, the treatment of FSGS, which was often refractory, varied, and the effectiveness of rituximab in FSGS was controversial [18]. Thus, an adrenocor- ticotropic hormone gel was recommended, which might be effective for refractory FSGS [19]. Based on our findings, following a diagnosis of MCD or FSGS confirmed using PEC marker with 1 kidney biopsy, we may be able to decide on the appropriate treatment at an early stage.

Nevertheless, our study has several limitations. First, it was a single-center, retrospective, case-cohort study in which strategic bias, especially for the indication of kidney biopsy, may have existed. Our institution has strict guidelines for repeat biopsy. Therefore, the actual number of PAX8+ MCD cases may be actually higher than we have reported. Smeets et al. [6] reported that 14 of 57 single biopsies (25\%) had MCD with PEC+ marker. Second, we did not confirm that PAX8 is the only PEC marker feasible. However, we chose PAX8 because PAX8 is a cell nucleolar marker and sensitive to active PEC. Third, our study did not include an adequate sample size, and data were missing (e.g., proteinuria). Moreover, we consider no difference in proteinuria between both groups because no differences in serum albumin were found. Fourth, our study did not indicate the effect of treatment. As a longterm study with involvement of many nephrologists, treatment strategy varied. It has been reported that early relapse of FSGS after transplantation is not associated with FSGS lesions [20]. In vitro, corticosteroids prevent podocyte apoptosis induced by puromycin, an antibiotic that causes the nephrotic syndrome [21]. However, transplant patients were not included in our study. In addition, our initial treatment was the same as that long ago in Japan (oral glucocorticoids with steroid pulse therapy). Therefore, there may have been little influence of treatment.

\section{Conclusion}

We confirmed the diagnosis of MCD or FSGS using results of repeat biopsy and PAX8, a PEC marker. We observed that more PAX8+ patients were finally diagnosed with FSGS than PAX8- patients. Considering our results, PEC marker staining in patients with the nephrotic syndrome, e.g., MCD, may help to diagnose FSGS. In addition, the diagnosis based on PEC marker evaluation may be useful in the selection of initial treatment for the nephrotic syndrome.

\section{Acknowledgment}

The authors thank Mami Ohori, Yoshiko Ono, and Mie Tagaya for their technical support. 


\section{Statement of Ethics}

This study is a retrospective study carried out by the opt-out method of our hospital. This study was approved by the Institutional Committee on Human Research of our institution (approval No. 4059).

\section{Disclosure Statement}

The authors have no conflicts of interest to declare.

\section{Funding Sources}

The study received no financial support.

\section{Author Contributions}

T.S., S.W., and D.I. designed the study. T.S. and K.K. examined patient data. T.U. provided technical support. T.S., T.U., W.H., S.W., K.Y., M.N., D.I., S.S., and Y.S. participated in the interpretation of data. T.S., T.U., D.I., and J.K. made the pathological diagnosis. T.U. and N.I. revised the manuscript. All authors have approved the final manuscript.

\section{References}

1 D’Agati VD, Kaskel FJ, Falk RJ. Focal segmental glomerulosclerosis. N Engl J Med. 2011 Dec;365(25):2398-411.

2 Rich AR. A hitherto undescribed vulnerability of the juxtamedullary glomeruli in lipoid nephrosis. Bull Johns Hopkins Hosp. 1957 Apr;100(4):173-86.

3 Ueno T, Kobayashi N, Nakayama M, Takashima Y, Ohse T, Pastan I, et al. Aberrant Notch1-dependent effects on glomerular parietal epithelial cells promotes collapsing focal segmental glomerulosclerosis with progressive podocyte loss. Kidney Int. 2013 Jun 83(6):1065-75

4 Sakamoto K, Ueno T, Kobayashi N, Hara S, Takashima Y, Pastan I, et al. The direction and role of phenotypic transition between podocytes and parietal epithelial cells in focal segmental glomerulosclerosis. Am J Physiol Renal Physiol. 2014 Jan;306(1):F98-104.

5 Fatima H, Moeller MJ, Smeets B, Yang HC, D'Agati VD, Alpers CE, et al. Parietal epithelial cell activation marker in early recurrence of FSGS in the transplant. Clin J Am Soc Nephrol. 2012 Nov;7(11):1852-8.

6 Smeets B, Stucker F, Wetzels J, Brocheriou I, Ronco P, Gröne HJ, et al. Detection of activated parietal epithelial cells on the glomerular tuft distinguishes early focal segmental glomerulosclerosis from minimal change disease. Am J Pathol. 2014 Dec;184(12): 3239-48.

7 Nishi S, Ubara Y, Utsunomiya Y, Okada K, Obata Y, Kai H, et al. Evidence-based clinical practice guidelines for nephrotic syndrome 2014. Clin Exp Nephrol. 2016 Jun; 20(3):342-70.
8 Gallon L, Leventhal J, Skaro A, Kanwar Y, Alvarado A. Resolution of recurrent focal segmental glomerulosclerosis after retransplantation. N Engl J Med. 2012 Apr;366(17):1648-9.

9 Wei C, El Hindi S, Li J, Fornoni A, Goes N, Sageshima J, et al. Circulating urokinase receptor as a cause of focal segmental glomerulosclerosis. Nat Med. 2011 Jul;17(8):952-60.

10 Maas RJ, Deegens JK, Smeets B, Moeller MJ, Wetzels JF. Minimal change disease and idiopathic FSGS: manifestations of the same disease. Nat Rev Nephrol. 2016 Dec;12(12):76876.

11 Ahmad H, Tejani A. Predictive value of repeat renal biopsies in children with nephrotic syndrome. Nephron. 2000 Apr;84(4):342-6.

12 Stadermann MB, Lilien MR, van de Kar NC, Monnens LA, Schröder CH. Is biopsy required prior to cyclophosphamide in steroidsensitive nephrotic syndrome? Clin Nephrol. 2003 Nov;60(5):315-7.

13 Webb NJ, Lewis MA, Iqbal J, Smart PJ, Lendon M, Postlethwaite RJ. Childhood steroidsensitive nephrotic syndrome: does the histology matter? Am J Kidney Dis. 1996 Apr;27(4): 484-8.

14 Korbet SM, Volpini KC, Whittier WL. Percutaneous renal biopsy of native kidneys: a single-center experience of 1,055 biopsies. Am J Nephrol. 2014;39(2):153-62.

15 Uezono S, Hara S, Sato Y, Komatsu H, Ikeda $\mathrm{N}$, Shimao $\mathrm{Y}$, et al. Renal biopsy in elderly patients: a clinicopathological analysis. Ren Fail. 2006;28(7):549-55.

16 Iijima K, Sako M, Nozu K, Mori R, Tuchida $\mathrm{N}$, Kamei K, et al.; Rituximab for Childhood-onset Refractory Nephrotic Syndrome
(RCRNS) Study Group. Rituximab for childhood-onset, complicated, frequently relapsing nephrotic syndrome or steroid-dependent nephrotic syndrome: a multicentre, double-blind, randomised, placebo-controlled trial. Lancet. 2014 Oct;384(9950):1273-81.

17 Katsuno T, Masuda T, Saito S, Kato N, Ishimoto T, Kato S, et al. Therapeutic efficacy of rituximab for the management of adult-onset steroid-dependent nephrotic syndrome: a retrospective study. Clin Exp Nephrol. 2019 Feb; 23(2):207-14.

18 Kronbichler A, Kerschbaum J, FernandezFresnedo G, Hoxha E, Kurschat CE, Busch M et al. Rituximab treatment for relapsing minimal change disease and focal segmental glomerulosclerosis: a systematic review. Am J Nephrol. 2014;39(4):322-30.

19 Hogan J, Bomback AS, Mehta K, Canetta PA, Rao MK, Appel GB, et al. Treatment of idiopathic FSGS with adrenocorticotropic hormone gel. Clin J Am Soc Nephrol. 2013 Dec; 8(12):2072-81.

20 Canaud G, Dion D, Zuber J, Gubler MC, Sberro R, Thervet E, et al. Recurrence of nephrotic syndrome after transplantation in a mixed population of children and adults: course of glomerular lesions and value of the Columbia classification of histological variants of focal and segmental glomerulosclerosis (FSGS). Nephrol Dial Transplant. 2010 Apr;25(4):1321-8.

21 Wada T, Pippin JW, Marshall CB, Griffin SV, Shankland SJ. Dexamethasone prevents podocyte apoptosis induced by puromycin aminonucleoside: role of p53 and Bcl-2-related family proteins. J Am Soc Nephrol. 2005 Sep;16(9):2615-25. 